\title{
Age-Related Gray and White Matter Changes in Normal Adult Brains
}

\author{
Farnaz Farokhian ${ }^{1,2, ~ \#, ~ C h u n l a n ~ Y a n g 1, ~ \#, ~ I m a n ~ B e h e s h t i ², ~, ~ *, ~ H i r o s h i ~ M a t s u d a ~}{ }^{2}$, Shuicai Wu ${ }^{1, *}$ \\ ${ }^{1}$ College of Life Science and Bioengineering, Beijing University of Technology, Beijing, 100022, China \\ ${ }^{2}$ Integrative Brain Imaging Center, National Center of Neurology and Psychiatry, Kodaira, Tokyo Japan \\ \#These authors equally contribute this work.
}

[Received March 15, 2017; Revised April 30 2017; Accepted May 2, 2017]

\begin{abstract}
Normal aging is associated with both structural changes in many brain regions and functional declines in several cognitive domains with advancing age. Advanced neuroimaging techniques enable explorative analyses of structural alterations that can be used as assessments of such age-related changes. Here we used voxel-based morphometry (VBM) to investigate regional and global brain volume differences among four groups of healthy adults from the IXI Dataset: older females (OF, mean age $68.35 \mathrm{yrs}$; $\mathrm{n}=69$ ), older males (OM, 68.43 yrs; $n=66$ ), young females (YF, 27.09 yrs; $n=71$ ), and young males (YM, 27.91 yrs; $n=71$ ), using 3D T1-weighted MRI data. At the global level, we investigated the influence of age and gender on brain volumes using a two-way analysis of variance. With respect to gender, we used the Pearson correlation to investigate global brain volume alterations due to age in the older and young groups. At the regional level, we used a flexible factorial statistical test to compare the means of gray matter (GM) and white matter (WM) volume alterations among the four groups. We observed different patterns in both the global and regional GM and WM alterations in the young and older groups with respect to gender. At the global level, we observed significant influences of age and gender on global brain volumes. At the regional level, the older subjects showed a widespread reduction in GM volume in regions of the frontal, insular, and cingulate cortices compared to the young subjects in both genders. Compared to the young subjects, the older subjects showed a widespread WM decline prominently in the thalamic radiations, in addition to increased WM in pericentral and occipital areas. Knowledge of these observed brain volume differences and changes may contribute to the elucidation of mechanisms underlying aging as well as age-related brain atrophy and disease.
\end{abstract}

Key words: aging, gender, MRI, voxel-based morphometry, brain volume

In the human brain, magnetic resonance imaging (MRI) has revealed morphometric brain changes due to development and aging [1-3]. Various studies show that there is a significant link between age and cognitive functions such as memory, language, attention, thinking, and executive skills [4-6]. The many investigations of the human brain over the past several decades have broadened our understanding of the brain and contributed to the monitoring of clinical treatment effects in many brain diseases in aging individuals, including Alzheimer's disease, Parkinson's disease, schizophrenia, dementia, depression, and multiple sclerosis. In addition, several research groups have investigated the effects of age on gray matter density [7], cortical thickness [8], white matter signal abnormalities [9], and alterations in structural and functional brain systems [10].

*Correspondence should be addressed to: Dr. Iman Beheshti, Integrative Brain Imaging Center, National Center of Neurology and Psychiatry, 4-1-1, Ogawahigashi-cho, Kodaira, Tokyo 187-8551, Japan. Email: Iman.beheshti@ ncnp.go.jp; Dr. Shuicai Wu, College of Life Science and Bioengineering, Beijing University of Technology, Beijing 100022, China. E-mail addresses: Wushuicai@bjut.edu.cn

Copyright: (C) 2017 Farokhian F et al. This is an open-access article distributed under the terms of the Creative Commons Attribution License, which permits unrestricted use, distribution, and reproduction in any medium, provided the original author and source are credited. 
In the present study, we investigated the differences in global and regional brain volume alterations between young and older adults with respect to gender. We used structural magnetic resonance imaging (sMRI) data because of its advantages (including good tissue contrast and excellent spatial resolution without radiation exposure), which are lacking in positron emission tomography and single photon emission computed tomography modalities. The location-specific measures derived from sMRI are influential biomarkers for assessments of brain volume alterations.

Voxel-based morphometry (VBM) was developed as an advanced method for performing group-wise comparisons of sMRI scans [11]. Briefly, VBM assesses whole-brain structures with voxel-by-voxel comparisons that can be used to compare tissue concentrations or volumes between subject groups to distinguish structural alterations [12]. The VBM technique has been widely used to assess the gray matter (GM) and white matter (WM) alterations in various brain diseases such as Alzheimer's disease [13-15], Parkinson's disease [16], and epilepsy [17]. We used the VBM technique in the present study to determine the overall and regional brain volume differences among healthy male and female young ( late 20s) and older ( late 60s) individuals.

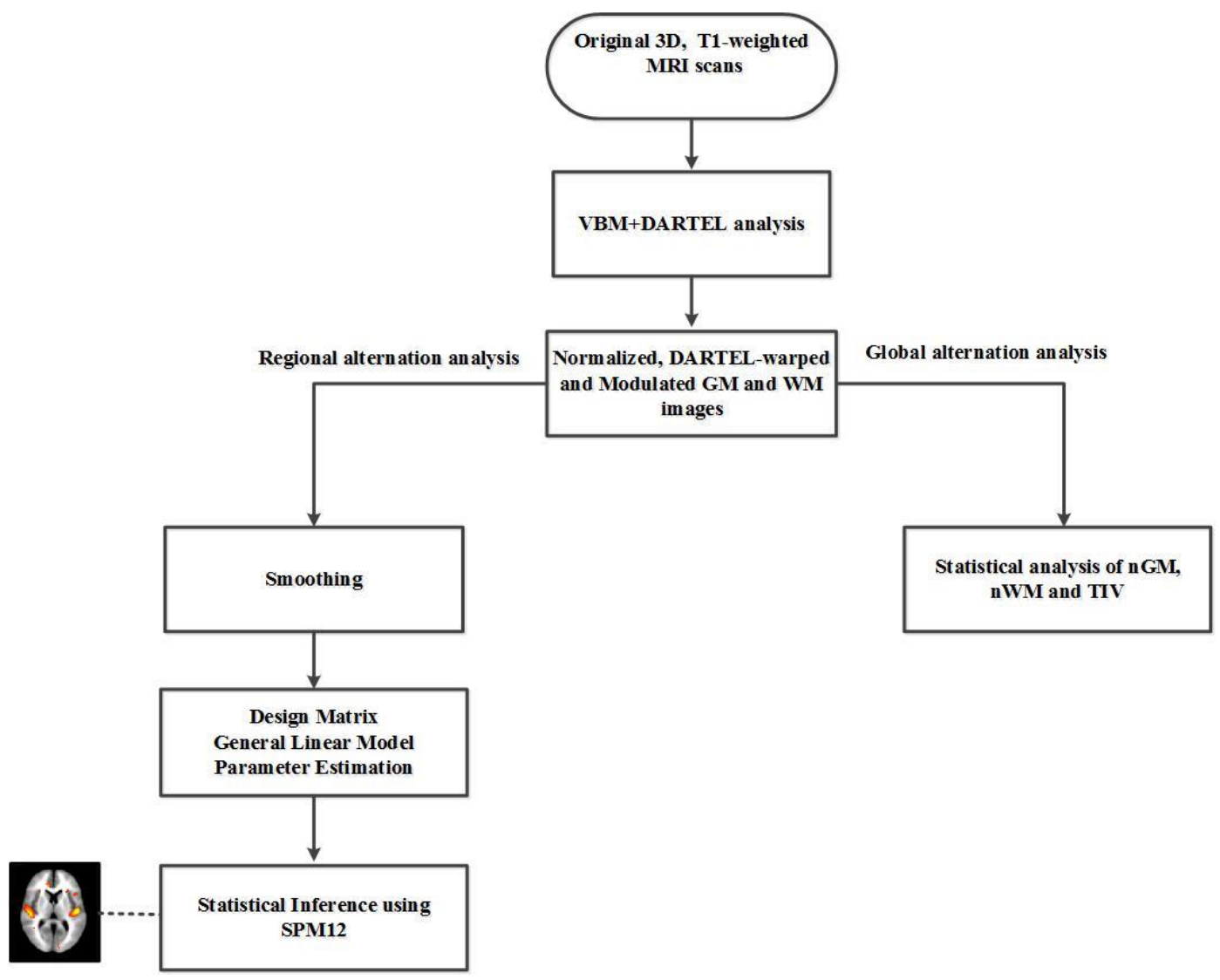

Figure 1. The general structure of proposed analysis procedure.

\section{MATERIALS AND METHODS}

\section{Image acquisition and subjects}

The data in the context of the present study were acquired from the publicly accessible IXI Dataset (braindevelopment.org/ixi-dataset/). The MRI scans were acquired from three sites with 1.5 and $3 \mathrm{~T}$ scanners $(\mathrm{FoV}=256 \mathrm{~mm} \times 256 \mathrm{~mm}$, matrix size $\left.=0.9375 \times 0.9375 \times 1.2 \mathrm{~mm}^{3}\right)$. Details of the IXI data and scan parameters are at: (http://biomedic.doc.ic.ac.uk/ brain-development/index.php? $\mathrm{n}=$ Main.Datasets).

As summarized in Table 1, we selected a total of 277 healthy subjects from the IXI Dataset and categorized them into the following four age/gender groups: older females (OF, mean age 68.35 yrs; $n=69$ ), older males (OM, 68.43 yrs; n=66), young females (YF, 27.09 yrs; $\mathrm{n}=71$ ), and young males (YM, 27.91 yrs; $\mathrm{n}=71$ ). There was no significant difference in age between the OF and OM groups or between the YF and YM groups. 
Table 1. The characteristics of the four groups of healthy subjects from the IXI Dataset

\begin{tabular}{lcccc}
\hline & Older females & Older males & Young females & Young males \\
& $(\mathrm{n}=69)$ & $(\mathrm{n}=66)$ & $(\mathrm{n}=71)$ & $(\mathrm{n}=71)$ \\
\hline Age $(\mathrm{yrs})$ & $68.35 \pm 5.80$ & $68.43 \pm 6.21$ & $27.09 \pm 3.59$ & $27.91 \pm 3.79$ \\
Range & $(60-86)$ & $(60-86)$ & $(20-34)$ & $(20-34)$ \\
\hline
\end{tabular}

All data are mean \pm standard deviation (SD).

\section{Methodology}

Figure 1 illustrates the major components of the methodology used in this study, including the acquisition of MRI scans, the VBM analysis, and the statistical analysis of brain volumes in the young and older groups with regard to gender.

The MRI preprocessing was performed using the Computational Anatomy Toolbox (CAT12; http://dbm.neuro.uni-jena.de/cat) [18] and Statistical Parameter Mapping (SPM) software ver. 12 (http://www.fil.ion.ucl.ac.uk/spm). Briefly, all MRI scans were segmented into WM, GM, and cerebrospinal fluid (CSF) components using the unified segmentation model [19], and then modulated and normalized into a Montreal Neurological Institute (MNI) template.

To provide a more regional and nonlinear deformation and to increase the intersubjective alignment of the MRI scans, we applied the DARTEL approach in the spatial normalization stage. The DARTEL approach helps optimize the sensitivity of such analyses by using the Levenberg-Marquardt strategy [20]. In addition, the DARTEL approach can provide more precise spatial normalization to the template compared to the conventional algorithm [21-23]. The details of a comparison of the DARTEL approach and the standard registration methods were as described [24].

In this study, we used the GM and WM images. Finally, with the use of an 8-mm full width at half maximum (FWHM) Gaussian isotropic kernel, the segmented GM and WM images were spatially smoothed. The smoothed, modulated, DARTEL-warped and normalized GM and WM modalities were used for our statistical analysis through a flexible factorial in the SPM12 program. Regional GM and WM alterations were generated by a voxel-based analysis over the whole brain. The absolute threshold for masking was adjusted to 0.2 to avoid possible edge effects between GM and WM or CSF. Group comparisons were assessed with the use of a family-wise error (FWE) at a threshold of $p<0.05$, corrected for multiple comparisons; statistical significance was determined using an extent threshold of 100 adjacent voxels.
In addition, to explore the regional GM and WM differences, we identified the global brain tissue volumes in the two older subject groups versus the two young groups with regard to gender. In this manner, we calculated the absolute GM volume (GMV), the absolute WM volume (WMV), and the absolute CSF volume as well as the total intracranial volume (TIV) of each subject. The TIV was calculated as the sum of the GMV, WMV, and CSF volumes. In order to correct for variation in the subjects' head sizes, we also calculated the normalized GM (nGM) and normalized WM (nWM) by dividing the individual subjects' GMV and WMV values by each subject's respective TIV value.

\section{Statistical analysis}

To assess the influence of two independent variables (age, gender) on global brain variables, we performed a twoway analysis of variance (ANOVA). Two levels of age (young and older) and gender (female and male) were examined. Between-group differences in global brain variables among or between groups were examined by an ANOVA followed by Tukey's multiple comparison test. We used the Pearson correlation test to investigate the association between brain volume changes and age. All statistical analyses were performed using Statistical Package for Social Sciences software (SPSS, ver. 16.0; SPSS, Chicago, IL) with $p<0.05$ as the significance level.

\section{RESULTS}

\section{Global differences in brain volume}

Whole-brain analyses help provide a reliable indication of total brain volume differences (in accord with age and gender) which occur in the entire brain. As described above in Section 2.2, we calculated the nGM and nWM in order to correct for variations in head size. Here we report the statistical results related to the influence of age and gender on global brain tissue volumes (i.e., the nGM, nWM and TIV) as well as the correlations between age and global brain tissue volume differences with respect to gender. 

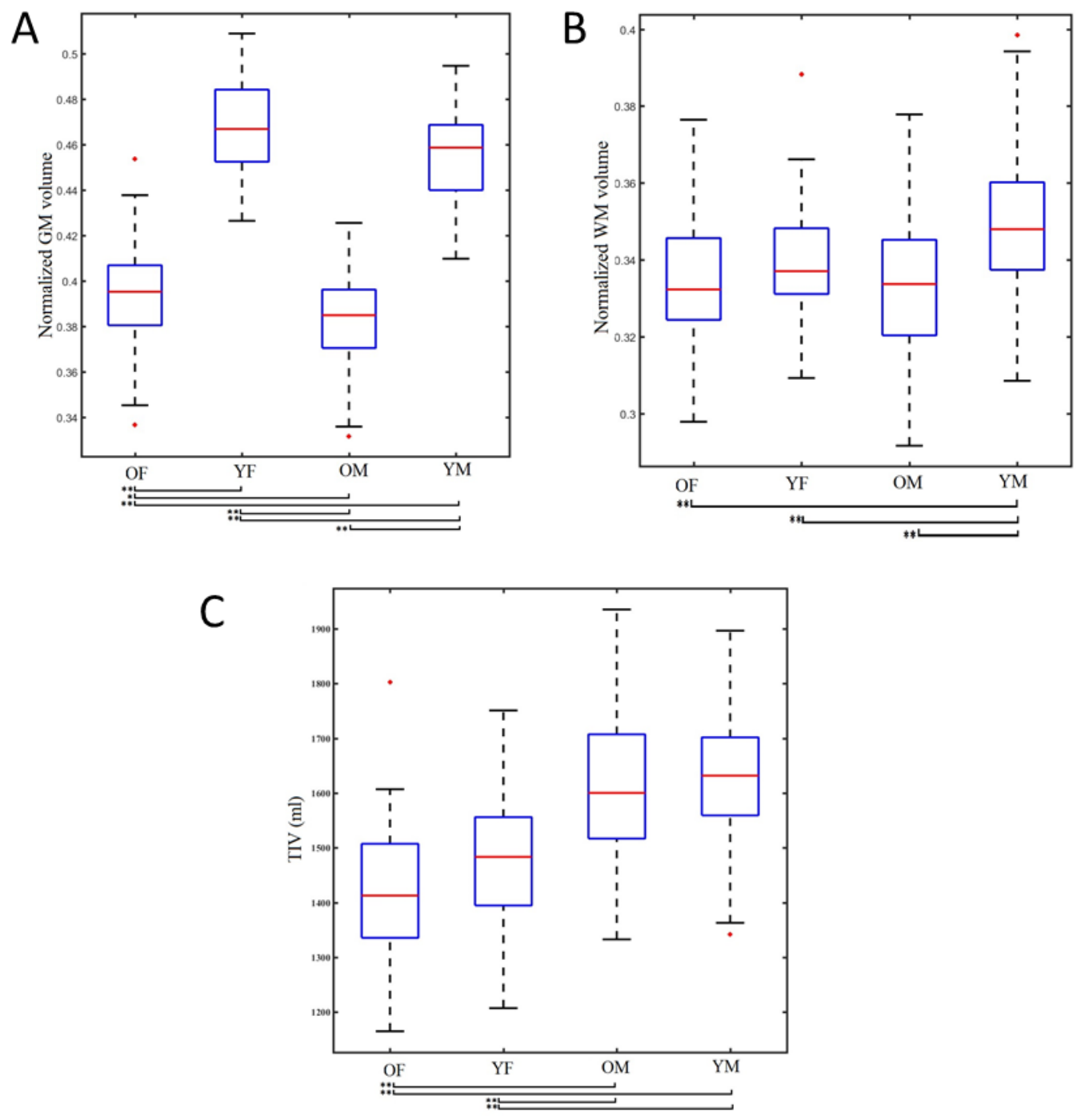

Figure 2. The box plots for the OF, YF, OM, and YM subjects. The box plots for the OF, YF, OM, and YM subjects' (A) normalized GM, (B) normalized WM and (C) total intracranial volume (TIV). Significance was determined by an ANOVA followed by Tukey's post-hoc test. * $p<0.05$, * $*<<0.001$.

The influence of age and gender on global brain volumes Table 2 presents the ranges of the whole-brain tissue volumes in the four subject groups. Regarding the nGM, the main effect of the age level yielded an F-ratio of $\mathrm{F}[1,273]=894.60, p<0.001$, indicating a significant difference in nGM between the young subjects (mean $[\mathrm{M}]=0.462, \quad \mathrm{SD}=0.020)$ and the older subjects $(\mathrm{M}=0.389, \mathrm{SD}=0.021)$. The main effect of gender yielded an F-ratio of $\mathrm{F}[1,273]=25.25, \quad p<0.001$, revealing a significant difference in $\mathrm{nGM}$ between the female $(\mathrm{M}=0.432, \mathrm{SD}=0.042)$ and male subjects $(\mathrm{M}=0.420, \mathrm{SD}=0.040)$. The interaction effect between age and gender on nGM was not significant: $\mathrm{F}[1,273]=0.079, p=0.77$.
In a direct comparison of the YF versus OF subjects, the average values of $\mathrm{nGM}$ were $0.468 \pm 0.022$ and $0.399 \pm 0.025$ (mean difference $[\mathrm{MD}]=0.073, p<0.001$ ), whereas the average nGM values of the $\mathrm{YM}$ and $\mathrm{OM}$ subjects were $0.455 \pm 0.012$ and $0.383 \pm 0.016$ $(\mathrm{MD}=0.072, p<0.001)$, respectively. Figure $2 \mathrm{~A}$ shows the normalized GM box plots for the four subject groups.

Regarding the $\mathrm{nWM}$, the main effect of age yielded an F-ratio of $\mathrm{F}[1,273]=30.39, p<0.001$, indicating a significant difference between the young subjects $(\mathrm{M}=0.344, \quad \mathrm{SD}=0.017)$ and the older subjects $(\mathrm{M}=0.333, \mathrm{SD}=0.016)$. The main effect of gender yielded an F-ratio of $\mathrm{F}[1,273]=5.90, \quad p<0.05$, demonstrating a significant difference in $\mathrm{nWM}$ between 
the females $(\mathrm{M}=0.336, \quad \mathrm{SD}=0.015)$ and males $(\mathrm{M}=0.341, \quad \mathrm{SD}=0.019)$. There was a significant interaction of age and gender on the nWM: $\mathrm{F}[1,273]=9.52, p<0.05$.

In our direct comparison of the YM and OM subjects, the groups' average nWM values were $0.349 \pm 0.013$ and $0.332 \pm 0.017(\mathrm{MD}=0.017, p<0.01)$, respectively. However, there was no significant difference in the average nWM values of the YF and OF groups. Figure $2 b$ shows the normalized WM box plots of the four age $\times$ gender groups.

Regarding the TIV, the main effect of age showed an F-ratio of $\mathrm{F}[1,273]=5.54, p<0.05$, i.e., a significant difference between the young subjects $(M=1548.60$, $\mathrm{SD}=142.19)$ and the older subjects $(\mathrm{M}=1512.40$,
$\mathrm{SD}=149.93)$. The main effect of gender had an F-ratio of $\mathrm{F}[1,273]=127.30, \quad p<0.001$, revealing a significant difference in TIV between the female $(M=1449.80$, $\mathrm{SD}=119.08)$ and male subjects $(\mathrm{M}=1613.90$, $\mathrm{SD}=124.77)$. The interaction effect was not significant: $\mathrm{F}[1,273]=0.78, p=0.37$.

In our direct comparisons between the YF versus OF subjects and the YM versus OM subjects, there were no significant differences in the average of TIV values. This absence of significant differences in TIV showed that the two female groups (YF and $\mathrm{OF}$ ) were comparable with respect to head size, as were the two male groups (YM and $\mathrm{OM}$ ). Figure $2 \mathrm{c}$ shows the TIV box plot for each group.

Table 2. The range of global volume measurements for the young and older female and male subjects

\begin{tabular}{ccccc}
\hline & $\begin{array}{c}\text { Older females } \\
(\mathrm{n}=69)\end{array}$ & $\begin{array}{c}\text { Young females } \\
(\mathrm{n}=66)\end{array}$ & $\begin{array}{c}\text { Older males } \\
(\mathrm{n}=71)\end{array}$ & $\begin{array}{c}\text { Young males } \\
(\mathrm{n}=71)\end{array}$ \\
\hline $\mathrm{GM}(\mathrm{ml})$ & $562.59 \pm 50.08$ & $689.66 \pm 59.77$ & $614.28 \pm 60.11$ & $739.58 \pm 56.97$ \\
$\mathrm{WM}(\mathrm{ml})$ & $476.56 \pm 44.65$ & $499.70 \pm 52.09$ & $533.98 \pm 57.17$ & $568.6425 \pm 53.71$ \\
$\mathrm{TIV}(\mathrm{ml})$ & $1425.90 \pm 108.93$ & $1473.12 \pm 124.58$ & $1602.91 \pm 132.94$ & $1624.20 \pm 116.68$ \\
$\mathrm{nGM}$ & $0.399 \pm 0.02$ & $0.468 \pm 0.02$ & $0.383 \pm 0.01$ & $0.455 \pm 0.01$ \\
$\mathrm{nWM}$ & $0.334 \pm 0.01$ & $0.339 \pm 0.01$ & $0.332 \pm 0.01$ & $0.349 \pm 0.01$ \\
\hline $\begin{array}{l}\text { All data are mean } \pm \text { SD. GM: gray matter; WM: white matter; TIV: total intracranial volume; nGM: normalized gray matter; } \\
\mathrm{nWM} \text { : normalized white matter. }\end{array}$
\end{tabular}

The correlation between the global brain tissue volume changes and age

To determine the effect of age on brain tissue volume changes, we estimated the correlation between age and global brain tissue volumes among the subject groups. Figure $3 \mathrm{a}-\mathrm{f}$ illustrates the results of the correlation analysis of nGM, nWM, and TIV values in accord with age and gender.

Regarding the nGM (Fig. 3A, B), there was a strong negative correlation between age and nGMV in the young subjects (females: $r(\mathrm{n}=71)=-0.42, p<0.001$; males: $r$ $(\mathrm{n}=71)=-0.33, \quad p<0.001)$ and in the older subjects (females: $r \quad(\mathrm{n}=69)=-0.60, \quad p<0.001 ;$ males: $r$ $(\mathrm{n}=66)=-0.58, p<0.001)$. In the comparison of the $\mathrm{nGM}$ changes, the statistical analysis revealed a significant linear reduction with age in the older subjects as well as in the young subjects. In comparison to the males, this decrease was steeper in the females in both the older and young groups.

Regarding the nWM (Fig. 3C, D), we observed a weak interaction between $\mathrm{nWM}$ and age in the young subjects (females: $r \quad(71)=0.03, p=\mathrm{ns}$; males: $r$ $(71)=0.15, p=n s)$. Notably, the Pearson correlation test revealed a significant negative interaction between $\mathrm{nWM}$ and age in the older subjects (females: $r(69)=-0.25$, $p<0.05$; males: $r(66)=-0.62, p<0.001)$, and this decrease was significantly steeper in the males compared to the females. There was no significant relationship between TIV and age in the various groups ( $p=$ ns) (Fig. 3E,F). 
A

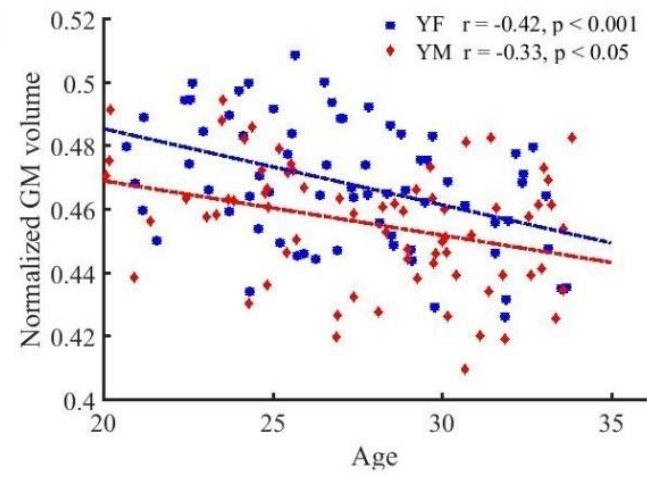

$\mathrm{C}$
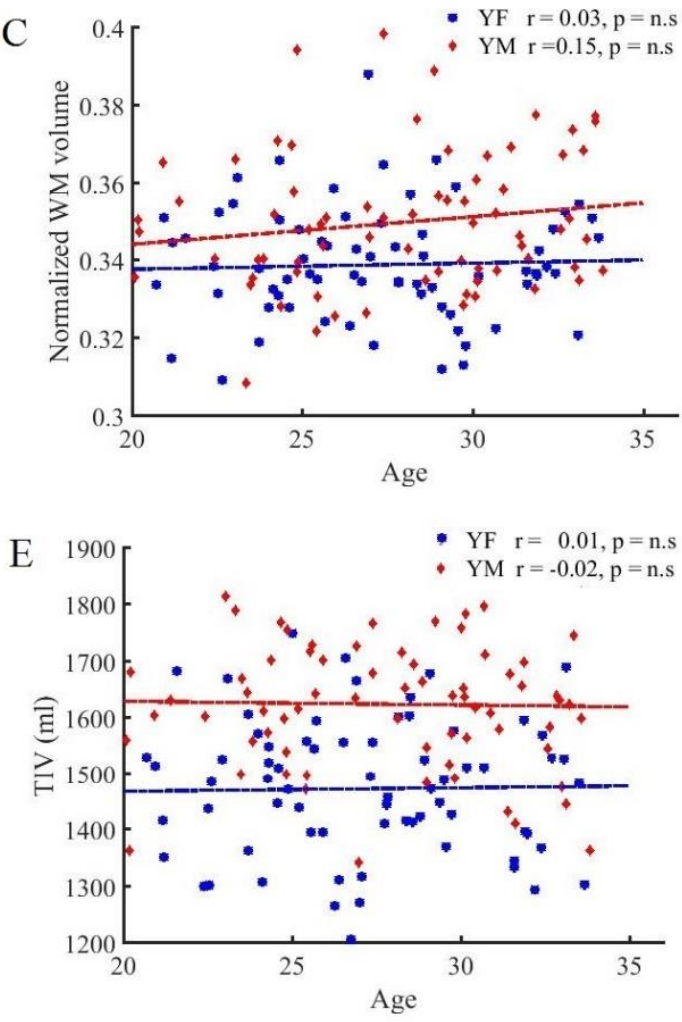
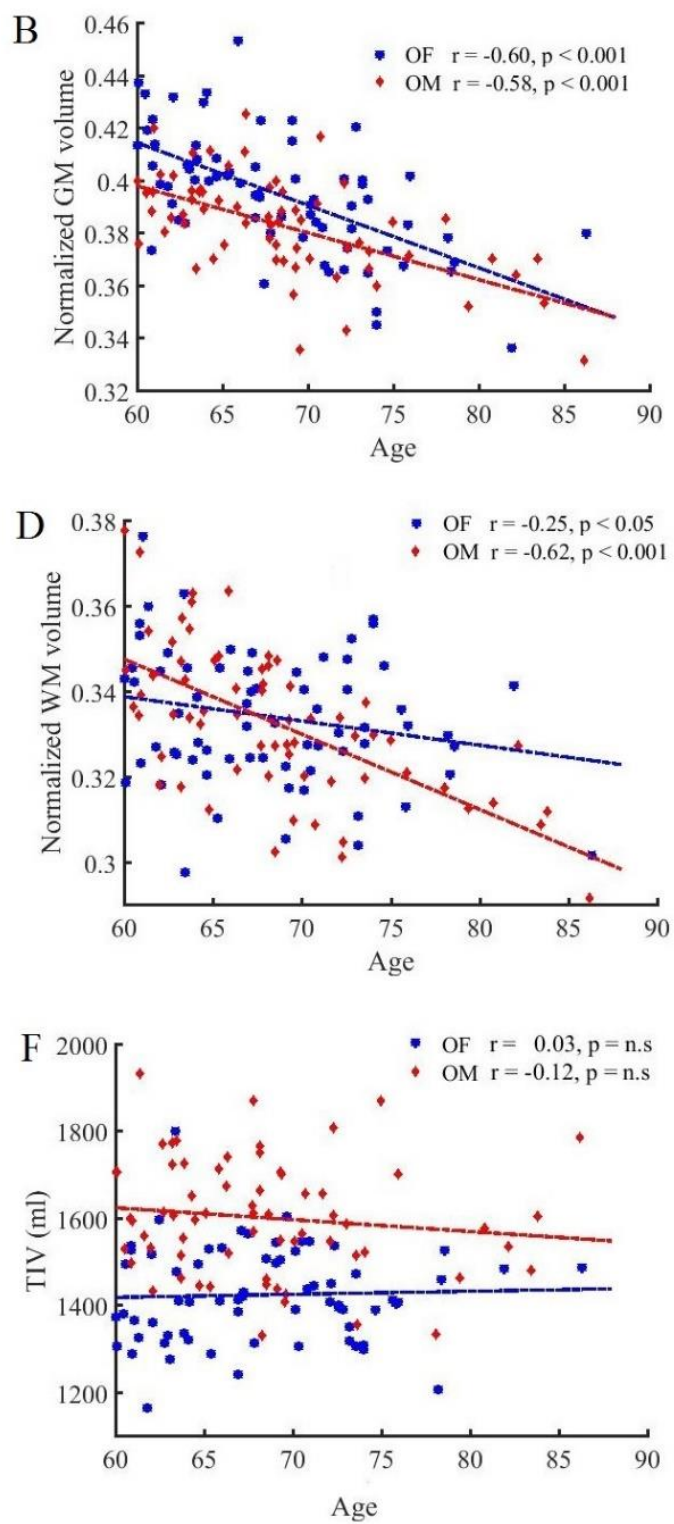

Figure 3. Plots of brain volumes vs. age in the young and older groups with respect to gender. (A) normalized GMV in the young subjects, (B) normalized GMV in the older subjects, (C) normalized WMV in the young subjects, (D) normalized WMV in the older subjects, (E) TIV in the young subjects, and (F) TIV in the older subjects.

\section{Regional differences in brain volume}

\section{GM regional differences}

Figure 4 shows the results of the group comparison of GM volume between the older and young subjects with respect to gender as well as the F-test results for the four groups. The VBM analysis revealed a diffuse and age-related reduction in the GM volume prominently in the frontal, insular, and cingulate cortex in both genders. In contrast, the occipitoparietal areas, medial temporal structures, and subcortical gray matter were relatively spared the age- related reduction in both genders. No significant interaction of age $\times$ gender regarding the reginal GM volume was observed. The reverse contrast showed no significant GMV reduction in the young subjects compared with the aging subjects.

\section{WM regional differences}

Figure 5 illustrates the results of the group comparisons of WM volume between the older and young subjects by gender as well as the F-test results among the four groups. 
The VBM analysis revealed an age-related decline in the $\mathrm{WM}$ volume, prominently in the thalamic radiations. In contrast, there was an increase in WM volume in the paracentral and occipital areas in both the female and male subjects. No significant interaction of age $\times$ gender was observed regarding reginal WM volume.

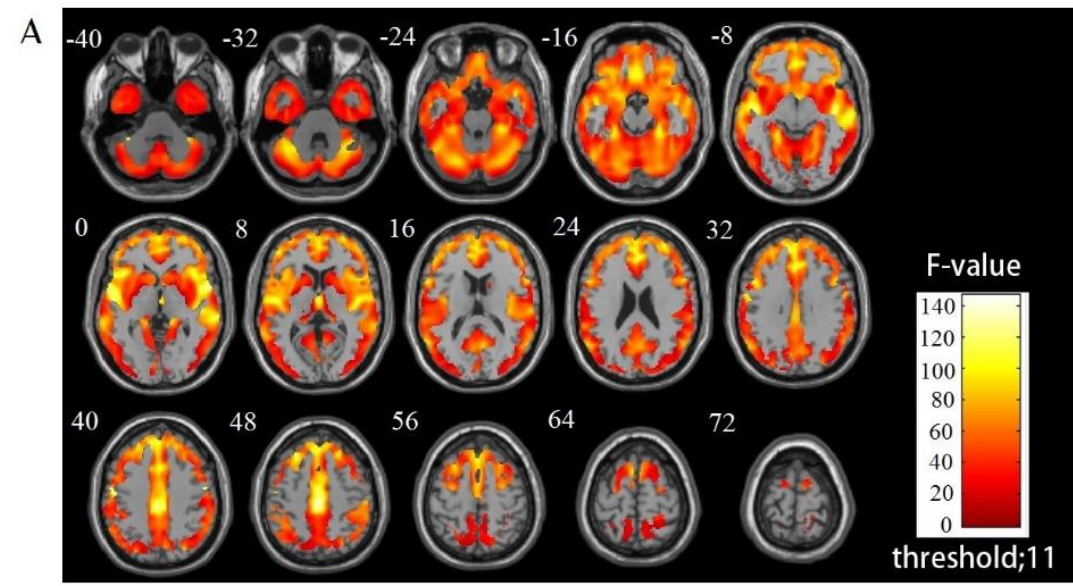

B

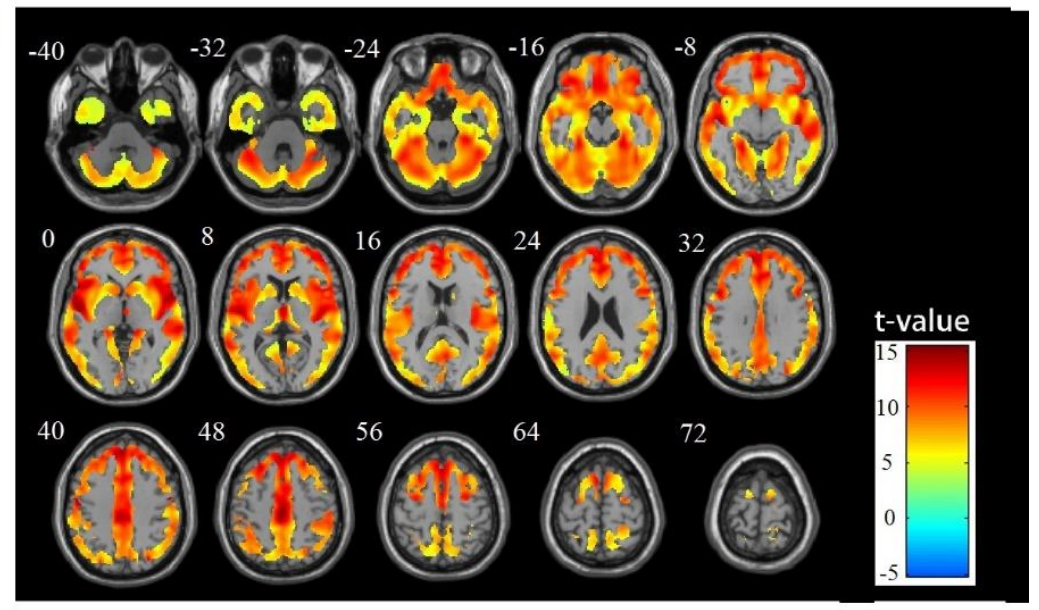

$\mathrm{C}$

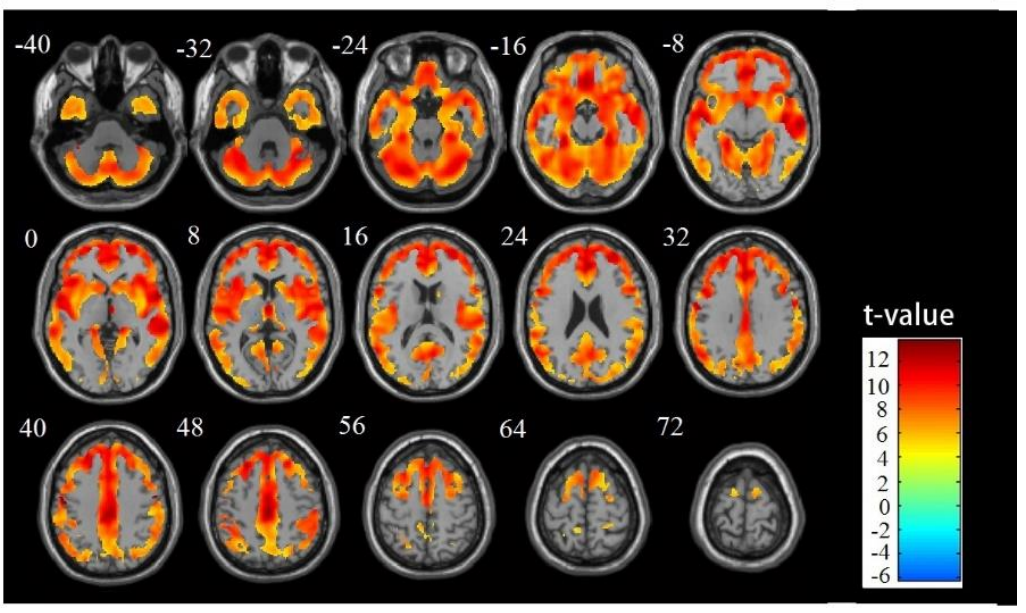

Figure 4. Group comparisons of GM volume alterations by VBM using SPM12 and DARTEL (FWE corrected at $p<0.05$ with extend threshold $K=100$ ). (A) F-test results for the four groups, (B) OF vs. YF, and (C) OM vs. YM. Warm and cool color scales show negative and positive correlations with age and volume, respectively 


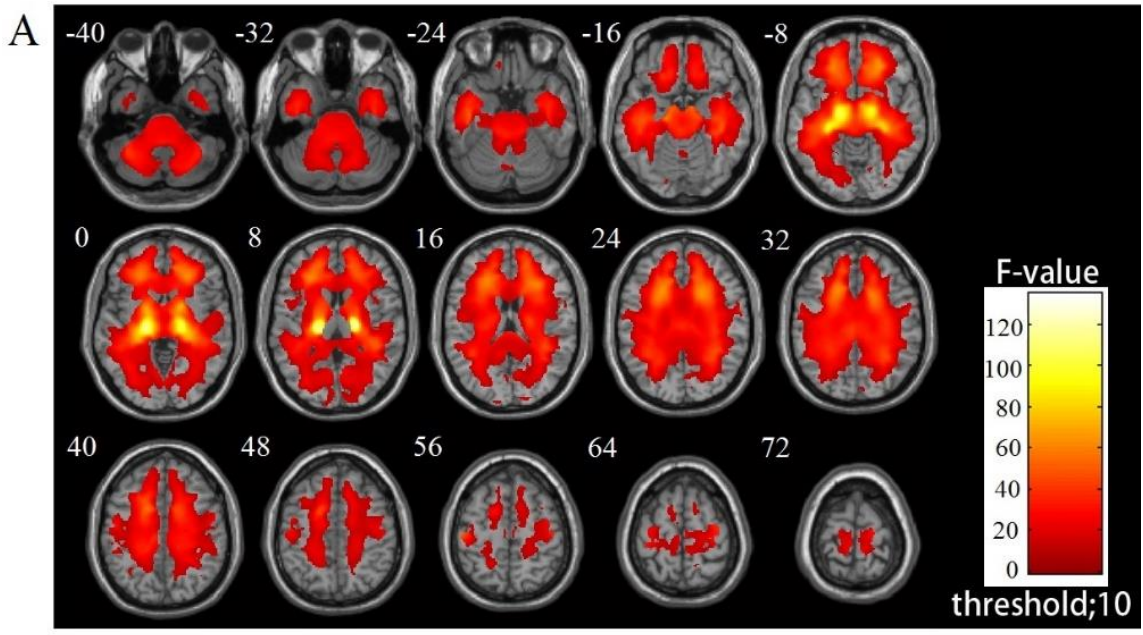

B

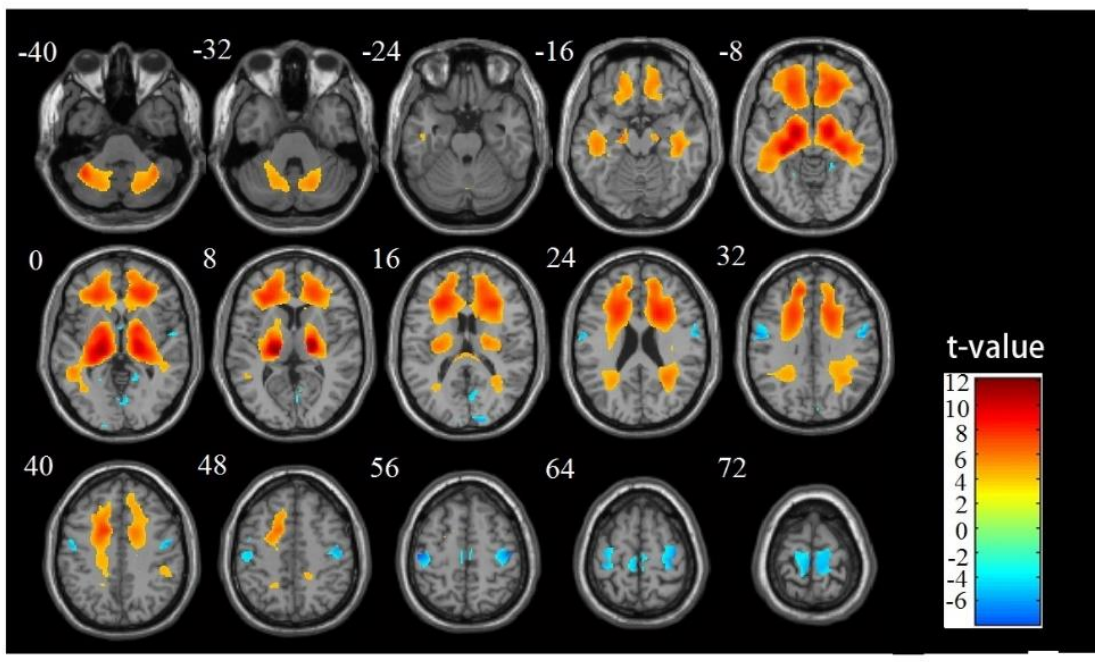

$\mathrm{C}$

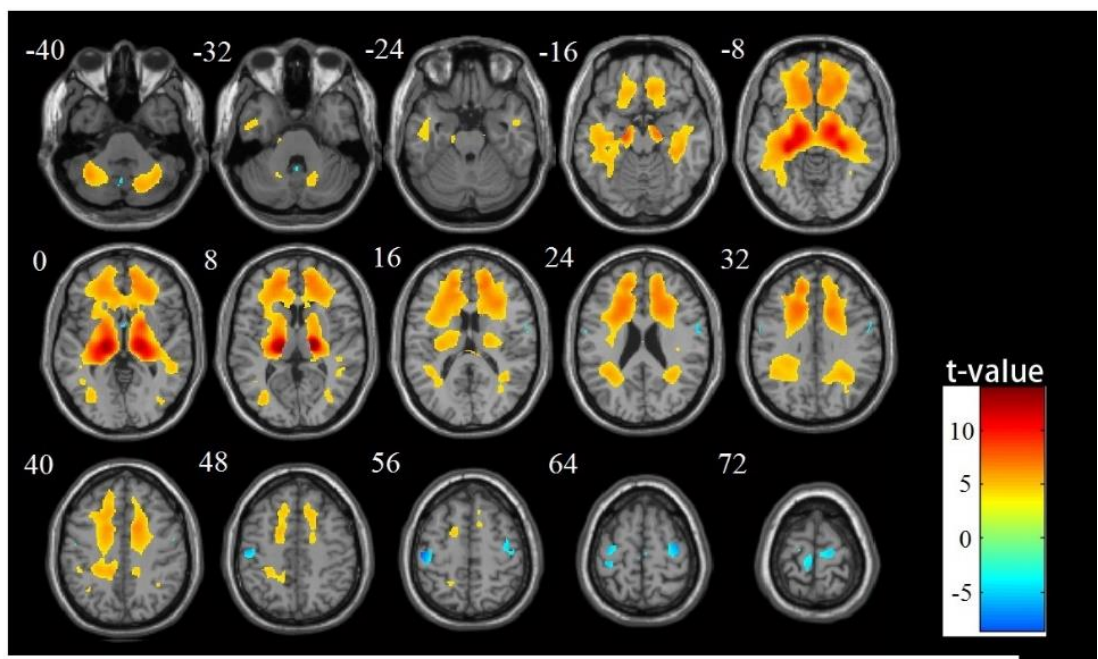

Figure 5. Group comparisons of WM volume alterations by VBM using SPM12 and DARTEL (FWE corrected at $p<0.05$ with extend threshold $\mathrm{K}=\mathbf{1 0 0}$ ). (A) F-test results of the four groups, (B) OF vs. YF, and (C) OM vs. YM. Warm and cool color scales show negative and positive correlations with age and volume, respectively. 


\section{DISCUSSION}

Our present findings indicate significant differences in the regional and global brain tissue volumes between our young and older subjects with respect to their gender. Further investigations of brain tissue volume differences between young and older individuals could lead to a better understanding of the normal aging process and clarify the pathology of age-associated neurological diseases. Several studies investigated the influence of aging on brain structures over time [8, 25-30]. Our present findings showed different global and regional patterns of GM and WM differences in healthy young and older individuals with respect to gender, as discussed in detail below.

\section{GM differences}

In our global GM analysis, the two-way ANOVA revealed significant influences of age and gender on global nGM volumes, in that the young subjects had superior total normalized GM volumes compared to the older subjects (i.e., YF and YM vs. OF and OM), and the female subjects showed higher nGM compared to the males (YF and OF vs. YM and $\mathrm{OM}$ ).

In the direct comparisons between the YF and OF subjects and between the YM and OM subjects, we observed superior total nGM volumes in the young females and young males compared to the older females and aging males, respectively (Fig. 2A). In addition, the correlation test showed that the normalized GM volume decreased linearly with age; this decrease was steeper in the females not only among the older subjects but also in the young group (Fig. 3A, B). Regarding the regional GM changes, we observed significant GM volume reductions in the frontal, insular, and cingulate cortices and a preservation of GM volume reductions in the occipitoparietal areas and subcortical regions among the older subjects compared to the young subjects of both genders.

These findings are generally in line with those of other studies which reported a linear negative association between GM volume and age for most cortical regions, prominently in the frontal and insular areas [26, 29-31]. Our results are also in agreement with other studies that demonstrated a preservation of the GM volume in specific structures (such as limbic and paralimbic brain structures) over the aging process[29, 31-33]. Consequently, it can be hypothesized that there are no significant alterations in limbic structures in older individuals with advancing age unless the alterations are due to specific neuropathological processes such as those related to cerebrovascular disease [34] or Alzheimer's disease [35].

\section{WM differences}

In our global WM analysis, the two-way ANOVA showed significant influences of age and gender on global nWM volumes, in that the young subjects showed higher nWM volumes compared to the older subjects (i.e., YF and YM vs. OF and OM), and the males showed slightly higher nWM volumes compared to the females (YM and OM vs. YF and OF).

In our direct comparison of the YF versus OF and YM versus OM groups, we observed a significant difference in $\mathrm{nWM}$ volumes between the older and young subjects among the males. Conversely, the difference in normalized WM volumes between the older and young subjects among the females was not significant (Fig. 2b). The reason(s) for this discrepancy between the genders are not clear, but may be related to the influence of sex hormones [36, 37].

Our analyses also demonstrated that there was no significant correlation between nWM volume changes with age in the young subjects (Fig. 3C) of both genders. The correlation test showed a significant negative association between nWM volume changes with age only in the older group for both genders, and this was significantly steeper in the male subjects (Fig. 3D).

With respect to regional WM differences, we observed a widespread reduction of WM volume prominently in the thalamic radiations. In contrast to some studies of healthy aging which describe only a decrease of $\mathrm{WM}$ in normal aging [31, 38, 39], we also observed significant WM increases in the pericentral and occipital areas among our older subjects compared to the young subjects, of both genders. The reason for this increase may be due to the ongoing maturation of the white matter during normal aging [40]. Our findings are broadly consistent with previous studies $[20,40]$ that reported a linear negative WM volume reduction associated with advancing age in anterior thalamic radiations, the internal capsule, cerebral peduncle, cerebellum, and external capsule among older subjects and a slight WM volume increase in bilateral corona radiata in the older subjects.

In future research, a diffusion tensor imaging analysis [41] between young and aging subjects with respect to gender should be considered. Another priority will be to use an individual network analysis [42] to determine the structural brain network differences between young and aging individuals with the consideration of gender.

\section{Conclusion}

In conclusion, we assessed regional and global brain tissue volume differences by conducting a VBM analysis of healthy young and older subjects of both genders. Our 
statistical analyses revealed different patterns of ageassociated alterations in both gray and white matter volumes in the young and older subjects. On the global level, we examined the effects of age and gender on normalized gray and white matter volumes as well as the total intracranial volume. We also explored the association between brain tissue volume changes with age in young and older subjects with respect to gender. We investigated the regional gray and white matter volume changes that had occurred in the brains of healthy subjects with age compared to those of young subjects, in both genders. Our findings indicate that there is a significant effect of brain volume changes during the aging process. Thus, the knowledge of brain volume changes and differences may contribute to a better understanding of the roots of health and disease in the later stages of life [43, 44].

\section{Acknowledgments}

This work has been partially supported by project grants from Beijing Nova Program (xx2016120), National Natural Science Foundation of China (81101107, 31640035), Natural Science Foundation of Beijing (4162008) and Scientific Research Project of Beijing Educational Committee (SQKM201710005013). This work was also partly carried out under the Brain Mapping by Integrated Neuro technologies for Disease Studies (Brain/MINDS) project (grant number 16dm 0207017h0003), funded by the Japan Agency for Medical Research and Development (AMED).

\section{References}

[1] Sullivan EV, Pfefferbaum A (2007). Neuroradiological characterization of normal adult ageing. Br J Radiol, 80 Spec No 2: S99-108

[2] Galluzzi S, Beltramello A, Filippi M, Frisoni GB (2008). Aging. Neurological Sciences, 29: 296-300

[3] van der Kolk AG, Hendrikse J, Zwanenburg JJ, Visser F, Luijten PR (2013). Clinical applications of 7 T MRI in the brain. Eur J Radiol, 82: 708-718

[4] Riddle DR, editor. Brain aging: models, methods, and mechanisms. CRC Press; 2007

[5] Meusel L-A, Grady CL, Ebert PE, Anderson ND (2017). Brain-behavior relationships in source memory: Effects of age and memory ability. Cortex, inpresss

[6] Singh R (2017) Electrophysiological Ageing of the Brain: Ageing-Related Impairments in Neural and Cognitive Functions. In Topics in Biomedical Gerontology, Singapore: Springer, 291-301

[7] Sowell ER, Peterson BS, Thompson PM, Welcome SE, Henkenius AL, Toga AW (2003). Mapping cortical change across the human life span. Nat Neurosci, 6: 309315

[8] Fjell AM, Westlye LT, Amlien I, Espeseth T, Reinvang
I, Raz N, et al. (2009). High consistency of regional cortical thinning in aging across multiple samples. Cereb Cortex, 19: 2001-2012

[9] Lindemer ER, Greve DN, Fischl BR, Augustinack JC, Salat DH (2017). Regional staging of white matter signal abnormalities in aging and Alzheimer's disease. Neuroimage Clin, 14: 156-165

[10] Andrews-Hanna JR, Snyder AZ, Vincent JL, Lustig C, Head D, Raichle ME, et al. (2007). Disruption of largescale brain systems in advanced aging. Neuron, 56: 924935

[11] Ashburner J, Friston KJ (2000). Voxel-based morphometry--the methods. Neuroimage, 11: 805-821

[12] Ashburner J, Friston KJ (2001). Why voxel-based morphometry should be used. Neuroimage, 14: 12381243

[13] Bagepally BS, John JP, Varghese M, Halahalli HN, Kota L, Sivakumar PT, et al. (2013). Relationship of clinical and cognitive variables with brain morphometric abnormalities in Alzheimer's disease: a voxel based morphometric study using 3-tesla MRI. Aging Dis, 4: 235-243

[14] Vasconcelos Lde G, Jackowski AP, Oliveira MO, Flor YM, Bueno OF, Brucki SM (2011). Voxel-based morphometry findings in Alzheimer's disease: neuropsychiatric symptoms and disability correlations preliminary results. Clinics (Sao Paulo), 66: 1045-1050

[15] Hirata Y, Matsuda H, Nemoto K, Ohnishi T, Hirao K, Yamashita F, et al. (2005). Voxel-based morphometry to discriminate early Alzheimer's disease from controls. Neurosci Lett, 382: 269-274

[16] Summerfield C, Junque C, Tolosa E, Salgado-Pineda P, Gomez-Anson B, Marti MJ, et al. (2005). Structural brain changes in Parkinson disease with dementia: a voxel-based morphometry study. Arch Neurol, 62: 281285

[17] Keller SS, Roberts N (2008). Voxel-based morphometry of temporal lobe epilepsy: an introduction and review of the literature. Epilepsia, 49: 741-757

[18] Gaser C, Dahnke R (2016). CAT-A Computational Anatomy Toolbox for the Analysis of Structural MRI Data. HBM 2016: 336-348

[19] Ashburner J, Friston KJ (2005). Unified segmentation. Neuroimage, 26: 839-851

[20] Matsuda H (2013). Voxel-based Morphometry of Brain MRI in Normal Aging and Alzheimer's Disease. Aging Dis, 4: 29-37

[21] Matsuda H, Mizumura S, Nemoto K, Yamashita F, Imabayashi E, Sato N, et al. (2012). Automatic voxelbased morphometry of structural MRI by SPM8 plus diffeomorphic anatomic registration through exponentiated lie algebra improves the diagnosis of probable Alzheimer Disease. AJNR Am J Neuroradiol, 33: $1109-1114$

[22] Ashburner J (2007). A fast diffeomorphic image registration algorithm. Neuroimage, 38: 95-113

[23] Pereira JM, Xiong L, Acosta-Cabronero J, Pengas G, Williams GB, Nestor PJ (2010). Registration accuracy for VBM studies varies according to region and degenerative disease grouping. Neuroimage, 49: 2205- 


\section{5}

[24] Yassa MA, Stark CE (2009). A quantitative evaluation of cross-participant registration techniques for MRI studies of the medial temporal lobe. Neuroimage, 44: 319-327

[25] Salat DH, Lee SY, van der Kouwe AJ, Greve DN, Fischl B, Rosas HD (2009). Age-associated alterations in cortical gray and white matter signal intensity and gray to white matter contrast. Neuroimage, 48: 21-28

[26] Curiati PK, Tamashiro JH, Squarzoni P, Duran FL, Santos LC, Wajngarten M, et al. (2009). Brain structural variability due to aging and gender in cognitively healthy Elders: results from the Sao Paulo Ageing and Health study. AJNR Am J Neuroradiol, 30: 1850-1856

[27] Good CD, Johnsrude I, Ashburner J, Henson RN, Friston KJ, Frackowiak RS (2001). Cerebral asymmetry and the effects of sex and handedness on brain structure: a voxelbased morphometric analysis of 465 normal adult human brains. Neuroimage, 14: 685-700

[28] Ziegler DA, Piguet O, Salat DH, Prince K, Connally E, Corkin S (2010). Cognition in healthy aging is related to regional white matter integrity, but not cortical thickness. Neurobiol Aging, 31: 1912-1926

[29] Grieve SM, Clark CR, Williams LM, Peduto AJ, Gordon E (2005). Preservation of limbic and paralimbic structures in aging. Hum Brain Mapp, 25: 391-401

[30] Tisserand DJ, van Boxtel MP, Pruessner JC, Hofman P, Evans AC, Jolles J (2004). A voxel-based morphometric study to determine individual differences in gray matter density associated with age and cognitive change over time. Cereb Cortex, 14: 966-973

[31] Good CD, Johnsrude IS, Ashburner J, Henson RN, Friston KJ, Frackowiak RS (2001). A voxel-based morphometric study of ageing in 465 normal adult human brains. Neuroimage, 14: 21-36

[32] Terribilli D, Schaufelberger MS, Duran FL, Zanetti MV, Curiati PK, Menezes PR, et al. (2011). Age-related gray matter volume changes in the brain during non-elderly adulthood. Neurobiol Aging, 32: 354-368

[33] Kalpouzos G, Chetelat G, Baron JC, Landeau B, Mevel K, Godeau C, et al. (2009). Voxel-based mapping of brain gray matter volume and glucose metabolism profiles in normal aging. Neurobiol Aging, 30: 112-124

[34] Gianaros PJ, Greer PJ, Ryan CM, Jennings JR (2006).
Higher blood pressure predicts lower regional grey matter volume: Consequences on short-term information processing. Neuroimage, 31: 754-765

[35] Garrido GE, Furuie SS, Buchpiguel CA, Bottino CM, Almeida OP, Cid CG, et al. (2002). Relation between medial temporal atrophy and functional brain activity during memory processing in Alzheimer's disease: a combined MRI and SPECT study. J Neurol Neurosurg Psychiatry, 73: 508-516

[36] Cowell PE, Sluming VA, Wilkinson ID, Cezayirli E, Romanowski CA, Webb JA, et al. (2007). Effects of sex and age on regional prefrontal brain volume in two human cohorts. Eur J Neurosci, 25: 307-318

[37] Eberling JL, Wu C, Tong-Turnbeaugh R, Jagust WJ (2004). Estrogen- and tamoxifen-associated effects on brain structure and function. Neuroimage, 21: 364-371

[38] Smith CD, Chebrolu H, Wekstein DR, Schmitt FA, Markesbery WR (2007). Age and gender effects on human brain anatomy: a voxel-based morphometric study in healthy elderly. Neurobiol Aging, 28: 10751087

[39] Resnick SM, Pham DL, Kraut MA, Zonderman AB, Davatzikos C (2003). Longitudinal magnetic resonance imaging studies of older adults: a shrinking brain. $\mathrm{J}$ Neurosci, 23: 3295-3301

[40] Giorgio A, Santelli L, Tomassini V, Bosnell R, Smith S, De Stefano N, et al. (2010). Age-related changes in grey and white matter structure throughout adulthood. Neuroimage, 51: 943-951

[41] Le Bihan D, Mangin JF, Poupon C, Clark CA, Pappata S, Molko N, et al. (2001). Diffusion tensor imaging: concepts and applications. J Magn Reson Imaging, 13: 534-546

[42] Tijms BM, Series P, Willshaw DJ, Lawrie SM (2012). Similarity-based extraction of individual networks from gray matter MRI scans. Cereb Cortex, 22: 1530-1541

[43] Trojano M, Liguori M, Bosco Zimatore G, Bugarini R, Avolio C, Paolicelli D, et al. (2002). Age-related disability in multiple sclerosis. Ann Neurol, 51: 475-480

[44] Baquer NZ, Taha A, Kumar P, McLean P, Cowsik SM, Kale RK, et al. (2009). A metabolic and functional overview of brain aging linked to neurological disorders. Biogerontology, 10: 377-413 